\title{
FUNCTIONAL LASER TRIMMING OF AUTOMOTIVE ELECTRONICS
}

\author{
JUNJI MITOBE and CHRIS DAVISON
}

Teradyne K. K., Meguro-ku, Tokyo 153

\begin{abstract}
This paper discusses the use of laser trim systems for the trimming of automotive hybrids. Being high volume parts these devices are best trimmed in two stages; first, a passive trim of the resistor substrate and secondly, a functional trim. The requirements for such a functional trim are such as to demand a fully integrated system.
\end{abstract}

\section{INTRODUCTION}

The use of lasers to adjust the performance of hybrid circuits has added a new dimension to the electronics industry and created a new production technology. No longer is hybrid technology reserved to the laboratory or small run production. Hybrid construction can be chosen on its own merits for such high volume products as automotive ignition modules and telephone active filters.

With the use of laser trimming systems for these high volume parts it is necessary to consider issues such as productivity and reliability in addition to the performance of the test system instrumentation and the laser itself. As an example of functional laser trimming and its use in high volume production we have chosen to talk about automotive modules but we could equally well have chosen active filters or $\mathrm{d} / \mathrm{a}$ converters as other examples of extremely high volume parts.

In recent years, the hybrid circuit technique has been used in manufacturing electronic modules for the automobile that cover the range from voltage regulators and electronic ignitions to various sensors. Given the $\pm 20 \%$ tolerances usually associated with thick-film resistors, some sort of laser trimming is essential in order to meet performance goals.

\section{SEPARATE PASSIVE AND FUNCTIONAL TRIMMING}

The most economical way to trim automotive electronic modules is to first passively trim the resistors on a general purpose resistor-trim system and then actively trim them on a system specifically designed to recreate the actual automotive environment. Using two separate systems retains the high throughput advantages of passive trimming and permits more reliable active trimming.

The Teradyne W411 high-volume passive trim system and the A300 solid-state ignition adjust system offer the necessary capabilities for passive and active automotive trimming needs. The W411 utilizes a highspeed galvanometer beam positioner, a high-power YAG laser, a $7 \times 7$ in. step-and-repeat carrier, and specialized software to provide the throughput and reliability required for passive trimming.

The A300 configured for active trimming of ignition modules, simulates actual operation in an automotive environment so that the trim is relevant. For example, specialized high-current and high-voltage sources supply the required currents and voltages, and detectors measure peak voltages and currents. A waveform generator allows users to program any waveform into the device under test, and a universal interface permits user to interchange loading coils, without system modification.

A system such as the W411 first passively trims most of the resistors on a substrate to bring them within acceptable tolerances. Following this the remainder of the components such as capacitors, discrete diodes, and any silicon integrated circuits, are added to the device. The silicon integrated circuits are usually mounted in a flip-chip manner, again allowing freedom from vibration effects.

At this point, a system such as the A300 makes a final active trim to bring the unit into final specification. In the case of the voltage regulator, for example, an active trim is made for the final regulated voltage. During the past four or five years electronic voltage regu- 
lators have appeared in virtually all cars in the United States, Japan, and Europe.

In the case of sensors such as manifold pressure, airflow, or temperature, the final active trim is made on the device in order to calibrate the output of the device with respect to the parameter being sensed. The use of such sensors in automobiles is just beginning to come into widespread use spurred on by the need for centralized engine processors to handle such functions as fuel metering, pollution control, and the like.

\section{LASER TRIMMING OF IGNITION CIRCUITS}

Trimming ignition modules is a bit more complex. In the current version of electronic ignitions, the contact points are replaced by a Darlington transistor that can take the on-current of the coil (about 7 A) and can also withstand the inductive kick when the current is turned off at spark time. The combination of $7 \mathrm{~A}$ and up to $400 \mathrm{~V}$, in addition to the very high levels of reliability required, added to the heat and vibration present inside an automotive engine, tend to impose severe requirements on these output transistors. In order to limit the energy that the device must switch, a means of limiting the current in the output device must be found.

This is accomplished by having the electronic module regulate the maximum current at some preset value usually in the range of 5 to $7 \mathrm{~A}$. An active trim is made on the ignition device in order to ensure the correct current. Another requirement in addition to limiting the maximum energy that the device must switch is limiting the peak voltage to which the device is subject when the ignition coil is shut off, as this voltage can easily reach 500 or $600 \mathrm{~V}$. While it is possible to limit this voltage through clever design of the ignition coil, a more common technique is to employ a feedback clamp to the base of the output transistor. A second active trim is thus required to ensure that this peak voltage does not rise above allowable limits, usually in the neighbourhood of about $400 \mathrm{~V}$.

A further problem with ignition devices occurs because most inductive or Hall-effect type pickups produce an ON signal that is a constant "dwell" or constant percentage of an engine revolution. If this signal is used directly to switch the output transistor, at low speeds the device would spend most of its time in current limit, thus dangerously raising its power dissipation. A variety of techniques are used to change the constant dwell of a pickup device into an $\mathrm{ON}$ signal which is of constant-time duration. For some of the latest ignition devices, an additional trim is required to ensure that the $\mathrm{ON}$ time specifications are met.

The laser has proved indispensable in manufacturing low cost devices with the desired high precision. In the last ten years, laser trimming has moved from laboratory curiosity to a reliable flexible production tool. 

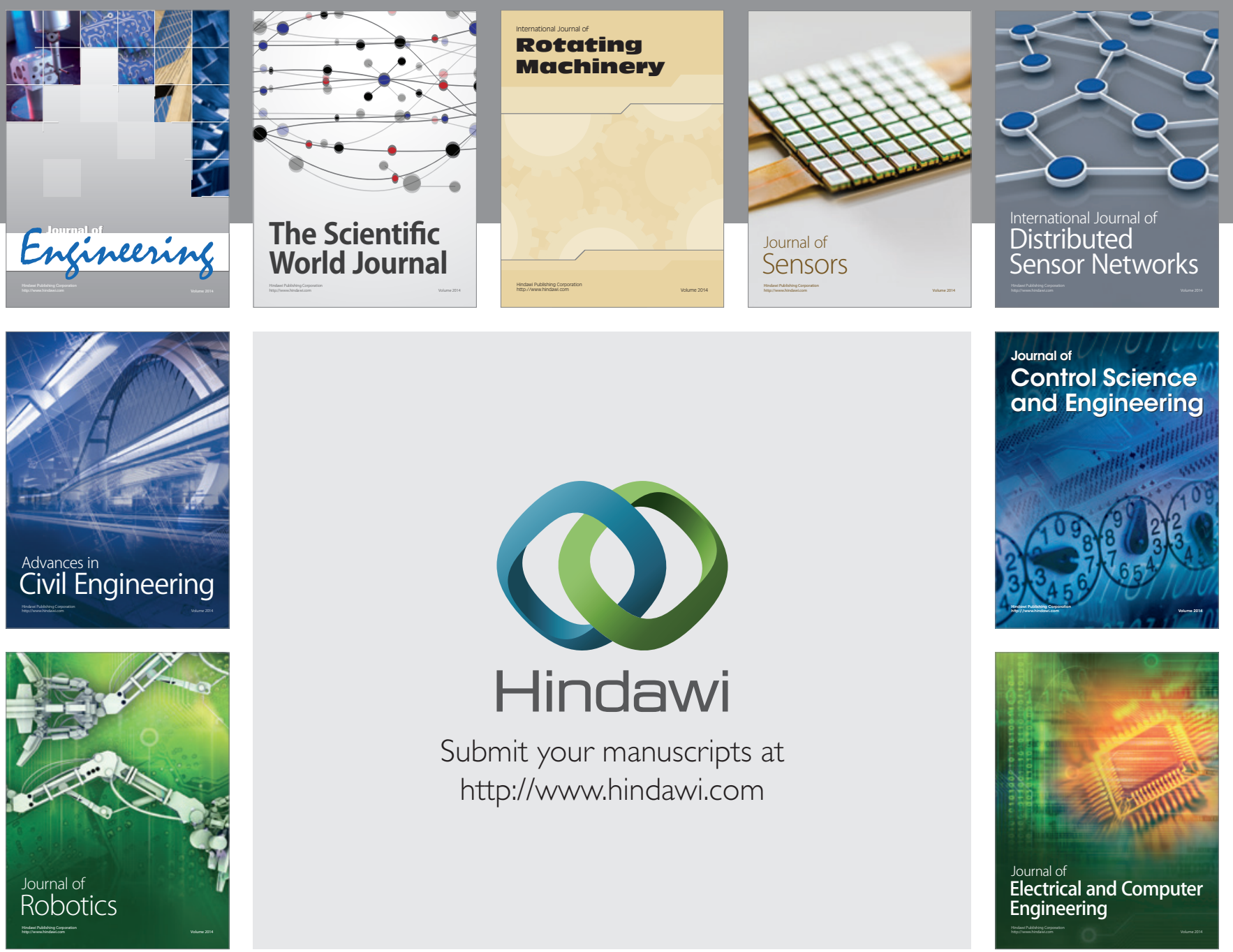

Submit your manuscripts at

http://www.hindawi.com
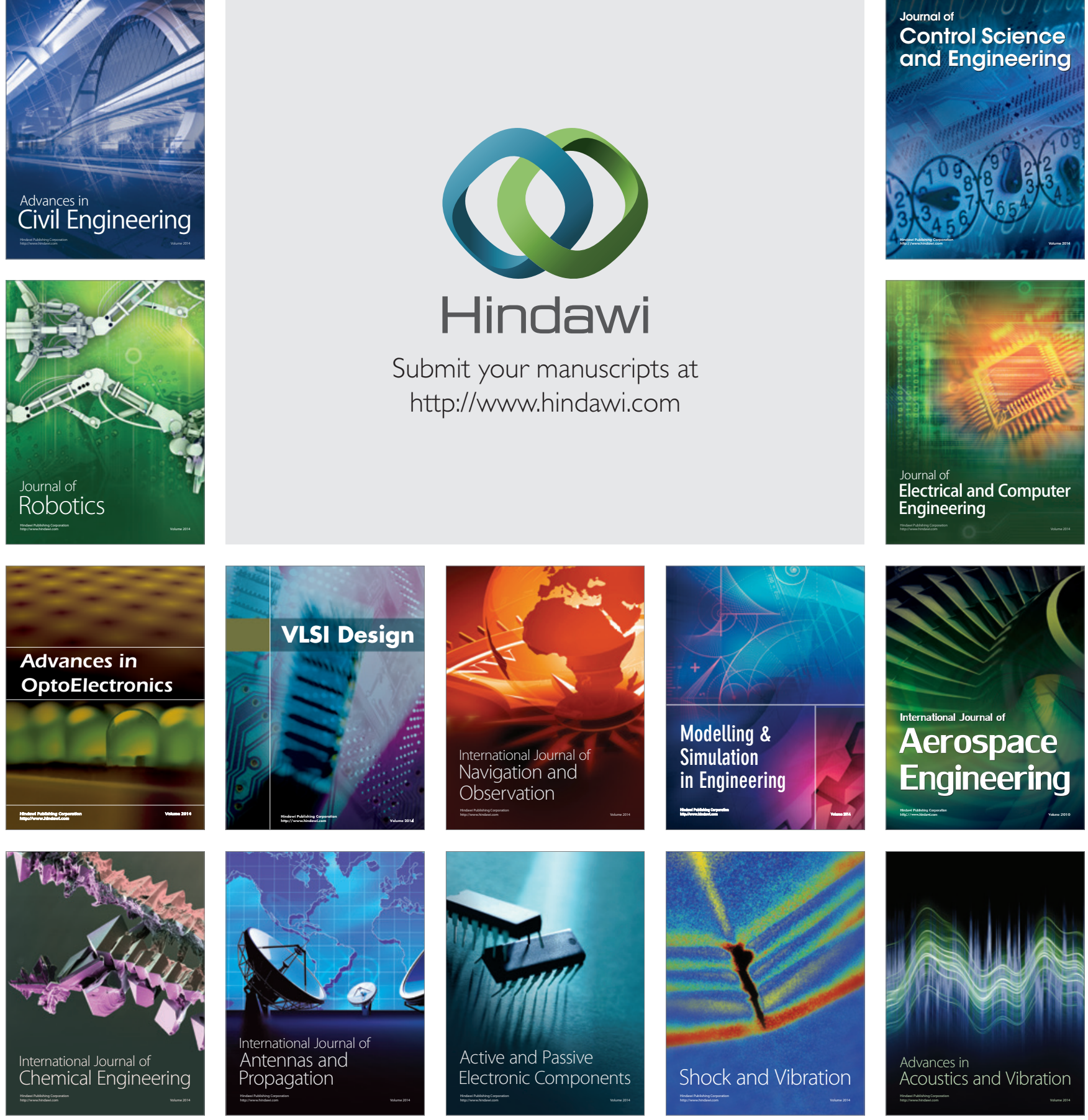